\title{
La marginalité et l'ambiguïté du personnage cynique en France. Une étude des romans (1990- 2010) de Frédéric Beigbeder
}

\author{
Louise Kari Mereau \\ Trinity College Dublin \\ Karimerl@tcd.Ie
}

Rebut: 23 de juny de 2018

Acceptat: 25 de juliol de 2018

RESUM

La marginalitat i l'ambigüetat del personatge cínic a França. Un estudi de les novel-les (1990-2010) de Frédéric Beigbeder

El cinisme, des dels seus orígens a la Antiga Grècia, és un corrent de pensament marginat ja que necessita sortir ja sigui per a denunciar-lo i millorar-lo, com volia Diògenes, o per a utilitzar els seus codis per al seu plaer personal, pràctica heretada dels llibertins durant el segle XVIII. En el segle XX, es percep al cínic com a un personatge arrogant, pedant i negatiu: se'l menysprea i critica sense tenir cap solució. S'exclou a si mateix tant com ho fa la societat.

En l'obra de Beigbeder, la marginalitat del personatge cínic és omnipresent i ambigua. Aquest cinisme es podria resumir en el següent: Com ser altruista sense ser individualista? Seria el cinisme un combat interior, sovint necessari que es llueix com a marginal per a trobar el seu lloc, paradoxalment en el si de la societat?

Aquests dos personatges, Marc i Octavi, son els alter-ego de l'autor, que sempre ha oscilllat entre l'èxit literari i comercial com la marginació cínica. Aquesta popularitat dels llibres de Beigbeder mostra la represa de l'atracció i la fascinació que la figura marginal del cínic exerceix a França des de la dècada dels 90 .

Paraules clau

Beigbeder, cinisme, marginalitat, ambigüitat, mundialització, sobre consum, altruisme, egocentrisme, individualisme. 


\section{RÉSUMÉ}

La marginalité et l'ambiguïté du personnage cynique en France. Une étude des romans (1990-2010) de Frédéric Beigbeder

Le cynisme, depuis son origine en Grèce antique, est un courant de pensée marginalisant puisqu'il nécessite que l'on s'extraie du monde, que ce soit pour le dénoncer et l'améliorer (comme Diogène le voulait) ou pour se servir de ses codes pour son plaisir personnel (pratique héritée des Libertins au $18^{\mathrm{e}}$ siècle). $\mathrm{Au} 20^{\mathrm{e}}$ siècle le cynique est perçu comme un personnage arrogant, pédant et négatif : il méprise et critique sans proposer de solution. Il s'exclut autant que la société l'exclut.

Dans l'œuvre de Beigbeder la marginalité du personnage cynique est omniprésente et ambiguë. Ce cynisme pourrait se résumer en la question suivante : 'comment être altruiste tout en restant individualiste ?' Le cynisme serait- il un combat intérieur - souvent nécessaire - brandi comme marginalité pour trouver sa place paradoxalement au sein de la société ?

Ses deux personnages, Marc et Octave, sont les alter-egos de l'auteur, qui toujours a oscillé entre succès littéraires et commerciaux et marginalisation cynique. Cette popularité des livres de Beigbeder montre la recrudescence de l'attrait et de la fascination que la figure marginale du cynique exerce en France depuis les années 1990.

MOTS-CLÉ

Beigbeder, cynisme, marginalité, ambiguïté, mondialisation, surconsommation, altruisme, égocentrisme, individualisme.

\section{RESUMEN}

La marginalidad y la ambigüedad del personaje cínico en Francia. Un estudio de las novelas (1990-2010) de Frédéric Beigbeder

El cinismo, desde sus orígenes en la Grecia antigua, es una corriente de pensamiento marginado puesto que necesita salir ya sea para denunciarlo y mejorarlo, como quería Diógenes, o para utilizar sus códigos para su placer personal, practica heredada de los libertinos en el siglo XVIII. En el siglo $\mathrm{XX}$, se percibe al cínico como un personaje arrogante, pedante y negativo: se le desprecia y critica sin tener ninguna solución. Se excluye así mismo tanto como lo hace la sociedad.

En la obra de Beigbeder, la marginalidad del personaje cínico es omnipresente y ambigua. Este cinismo podría resumirse en lo siguiente: ¿Cómo ser altruista sin ser individualista? ¿Sería el cinismo un combate interior, a menudo 
necesario que se luce como marginal para encontrar su lugar, paradójicamente en el seno de la sociedad?

Esos dos personajes, Marc y Octavio, son los alter-ego del autor, que siempre ha oscilado entre el éxito literario y comercial y la marginación cínica. Esta popularidad de los libros de Beigbeder muestra el recrudecimiento de la atracción y de la fascinación que la figura marginal del cínico ejerce en Francia desde la década de los 90 .

\section{Palabras clave}

Beigbeder, cinismo, marginalidad, ambigüedad, mundialización, consumo excesivo, altruismo, egocentrismo, individualismo.

\section{Abstract}

The marginality and the ambiguity of the cynical character in France. A study of Frederic Beigbeder's novels (1990-2010)

Cynicism, which originated in Ancient Greece, is a philosophical movement that promotes social self-exclusion and creates marginalisation since it requires an adherent to extract themselves from society, to denounce it, and to either improve it (as Diogenes would posit), or to use its codes and flaws for personal pleasure (as the Libertines have done since the 18th century). In the 20th century, the cynic is considered as arrogant, self-focused and negative: he despises and criticises without proposing a solution. He excludes himself as much as society excludes him.

In Beigbeder's works, the cynic character's marginality is omnipresent and ambiguous. This cynicism could be summarised as searching for the answer to the question of 'How to be altruistic while remaining individualist?' Cynicism to Beigbeder is the inner battle - often necessary - of using marginalisation, paradoxically, to find one's place in society.

His two characters, Marc and Octave, are the author's alter-egos, who constantly balance between literary and commercial successes and cynical marginalisation. The popularity of Beigbeder's books reveals an increase in the attraction towards and the fascination with the marginal figure of the cynic in France since the 1990s.

\section{KEYWORDS}

Beigbeder, cynicism, marginality, ambiguity, globalisation, over consumerism, altruism, egocentricity, individualism. 
Philosophie grecque développée par Diogène de Sinope ${ }^{1}$ en Grèce antique afin de révéler les travers de l'organisation humaine, le cynisme, au $18^{\text {ème }}$ siècle, se scinde en deux courants de pensée contradictoires : si la Révolution semble toujours user du cynisme comme Diogène le fit dans l'Antiquité - pour améliorer la société et permettre un accès au bonheur plus simple et plus efficace ${ }^{2}$ - ce siècle se révèle simultanément comme celui de de l'éveil de l'esprit libertin, et de l'esprit égocentré. ${ }^{3}$ Cette double forme du cynisme exerce une forte influence sur l'identité de la société française.

Les Lumières visèrent et lancèrent la modification de la société Française, de sa culture, son rapport à la religion et surtout de l'organisation de son économie politique, mais, comme le constate Sharon Stanley, dans son livre The French Enlightenment and Cynicism, les gens n'accueillent désormais plus les voyageurs chez eux sans les faire payer pour la couche et le diner. Si un paiement est attendu en échange d'un service, l'action n'est-elle plus altruiste ? ${ }^{4}$ Cette évolution dans le rapport à l'étranger montre la disparition, peu à peu, de valeurs, par exemple d'hospitalité que la société moderne du $20^{\text {ème }}$ siècle remplace par celles de l'esprit égocentré, marchand. ${ }^{5}$ Il est aussi important de noter l'importance du $19^{\text {ème }}$ siècle dans l'évolution de la philosophie cynique, comme le note Judith Skhlar dans After Utopia: The Decline of Political Faith : les romantiques ont perdu foi en l'optimisme des Lumières, et leur volonté d'améliorer la société. ${ }^{6}$ En effet, la population française constate que la Révolution n'a pas fonctionné comme elle l'espérait (La terreur instaurée par Robespierre, puis les périodes d'empires de Napoléon I avec ses guerres puis de Napoléon III avec le bousculement industriel, entrecoupées de révolutions et Républiques successives), et que les conditions de vie ne sont pas vraiment meilleures sous la République qui n'a pas été à l'abri de nouvelles guerres au $20^{\text {ème }}$ siècle alors que de nouvelles idéologies collectives s'étaient emparées

${ }^{1}$ J. BRUNSCHWIG \& Geoffrey E.R. LLOYD., A guide to Greek Thought, "The Pedagogical Challenge", de Marie-Odile GOULET-CAZÉ, traduit par Rita Guerlac et Anne Slack, Harvard University Press reference library, Belknap Press, 2000.

${ }^{2}$ Ibid. p. 327-328 et 333.

${ }^{3}$ Sharon A. STANLEY, The French Enlightenment and the Emergence of Modern Cynicism, "The Dark Side of Sociability, Philosophes and Libertines", Cambridge University Press, 2012, p. 75105 .

${ }^{4}$ Ibid. "Hermits and Cynics" et "The Dark Side of Sociability, Philosophes and Libertines".

${ }^{5}$ Comme le précise Daniel ROCHE, dans France in the Enlightenment, traduction d'Arthur Goldhammer, Cambridge, MA: Harvard University Press, 1998, p. 551.

${ }^{6}$ Judith SHKLAR, After Utopia: The Decline of Political Faith, Princeton, Princeton University Press, 1957, chapter 1. 
des esprits; cela marque le début de la perte de foi dans la politique. Judith Skhlar conclut que, de nos jours, de plus en plus de personnes - et de plus en plus jeunes - ressentent une sensation de désespoir politique, qui peut se traduire par une volonté de tout changer ou par un désintéressement.?

Après les années 1980 la société française comprend que la mondialisation et la société de consommation n'apportent pas le bonheur ; il y a dès lors un repli sur soi de la jeunesse qui devra peut-être murir avant de vouloir essayer résolument d'améliorer les conditions de la vie humaine. Fréderic Beigbeder fait partie de cette génération, qui a 20 ans dans les années 1980 et qui fut déçue par l'échec du sur-capitalisme. Comme il le dit lui-même, sa génération est celle qui a connu la fin de l'utopie universaliste qui s'est développée dans les années 1970 ; les années 1990-2000 sont la réalisation que la mondialisation ne rend personne plus heureux ou plus égal. Le cynique est quelqu'un qui décrit de façon divertissante l'horreur de la réalité.

Dès lors, à la fin du $20^{\text {ème }}$ siècle et à l'aube du $21^{\text {ème }}$ siècle, deux courants du cynisme s'affrontent, faisant de sa définition une tâche compliquée : soit l'on dénonce et l'on veut améliorer la société, soit on utilise les défauts de la société pour son plaisir personnel, ce qui conduit le plus souvent à un certain nihilisme. Dans les deux cas, le cynique doit s'extraire de la société pour mieux en appréhender les différentes facettes. Une condition est donc commune : le cynique a besoin de se marginaliser pour exister en tant que tel.

L'évolution du cynisme dans les œuvres de Frédéric Beigbeder souligne le caractère ambigu du personnage marginal qu'est le cynique contemporain. Ses personnages principaux - alter-egos de lui-même qui balancent entre succès littéraires et scandales publics - oscillent constamment entre le plaisir personnel et le bonheur sociétal. Marc Marronnier, jeune bourgeois arrogant qui ne veut pas s'agréger à la masse, manipule les esprits pour obtenir ce qu'il veut (Mémoires d'un jeune homme dérangé, Vacances dans le coma) tout en ne réussissant pas à être heureux en couple dans une société qui transforme l'amour en bien de consommation (L'Amour dure trois ans). Octave Parango (99 Francs) tente de se repentir de sa vie d'égocentrique débauché - son métier, publicitaire, lui confère l'impression et l'excuse d'être au-dessus de la masse, qu'il contrôle par le biais de la publicité, et son salaire exorbitant l'absout de ne rien respecter - pour lutter pour un monde meilleur, mais finit par retomber dans ses travers alors qu'il est exilé en Russie (Au secours pardon), ce qui ne l'empêche néanmoins pas de profiter du déplacement géographique pour mieux comprendre les dérives de la société de surconsommation.

\section{${ }^{7}$ Ibid.}


Dans cet article nous nous proposons d'étudier les différentes formes de marginalité des héros - ou anti-héros pourrait-on dire car leur destin n'est pas reluisant - beigbederiens afin d'approcher une possible définition du cynisme contemporain.

Pour la rigueur de notre étude, nous avons choisi d'évoquer en premier lieu la position sociale comme forme de marginalité. Si l'on pense aux personnages de Beigbeder, ils ont tous en commun de venir d'un milieu bourgeois et d'exercer un métier, sinon rare, au moins singulier: Marc Marronnier est écrivain et chroniqueur mondain nocturne, Octave Parango est d'abord concepteur-rédacteur dans une agence de publicité puis Talent Scout. Ainsi donc, même avant d'entrer dans l'histoire, le lecteur peut d'ores et déjà comprendre que le personnage principal ne sera pas ce que l'on peut appeler un monsieur-tout-le-monde ; une telle position a des privilèges et des inconvénients - comme le précise Marc, ironiquement ricanant. ${ }^{8}$ Le personnage principal des œuvres de Beigbeder, n'étant autre que différentes représentations de l'auteur à certaines étapes de sa vie, propose alors, sous la forme d'un monologue plus ou moins assumé, une description de sa vie en parallèle de celle du quidam habituel. Par exemple, dans la première saga, composée de trois romans : Mémoires d'un jeune homme dérangé, Vacances dans le coma et L'Amour dure trois ans, Marc Marronnier, narrateur intra-diégétique, écrit son histoire, vue par lui-même, une sorte de soliloque intérieur public. Il décrit ce qu'il fait, et pourquoi il le fait. La société française est vue depuis son unique point de vue. C'est d'ailleurs ce qu'Anne, son ex-épouse lui reprochera après un diner de retrouvailles, plutôt agité, « Marc, je vais te donner un tuyau pour tes rapports avec tes prochaines femmes. Il faut que tu apprennes à te mettre à leur place $»^{9}$ L'égocentrisme ne favorise pas les rapports humains, et encore moins le bonheur amoureux.

Cet égocentrisme, que l'on retrouve comme fil rouge au travers de tous les personnages principaux de Beigbeder, est au cœur de la marginalité chez Beigbeder. Par le biais de notre analyse, nous nous attacherons à montrer comment cet égocentrisme est à double tranchant, et comme il s'affilie à l'idée de rejet pour devenir cynisme - même s'il s'agit d'un cynisme ambigu.

\footnotetext{
${ }^{8}$ Frédéric BEIGBEDER, Mémoires d'un jeune homme dérangé, la petite Vermillon, Editions La Table Ronde, 2001, p. 20.

${ }^{9}$ Frédéric BEIGBEDER, L'Amour dure trois ans, Folio, Gallimard, 2005, p. 158.
} 


\section{Concept $1:$ Le rejet des autres}

Comme nous l'avons mentionné, Marc Marronnier ne semble penser qu'à lui-même. Pourtant, son attitude égocentrique révèle une précise analyse de la société moderne des années 1990. Il refuse d'en suivre les règles; pour se faire il doit les connaitre. Il veut être célèbre mais décrié, pour ce faire il doit d'abord être publié et susciter l'intérêt.

Jack Kerouac avait écrit dans Désolation Angels (1965) :

Qui sont-ils les hommes qui peuvent insulter les hommes ? Qui sont-ils les ricaneurs pantalonnés ? De quoi est-ce que je parle ? Je parle du désespoir humain, de l'incroyable solitude de la naissance et de la mort ténébreuse et je pose la question « En quoi cela prête-t-il à rire ? Comment peut-on faire le malin quand on est dans le hachoir à viande ? Qui nargue la misère ?10

Et Marc et ses amis titrent leur groupe « ricaneurs pantalonnés » pour expliquer, ou cultiver - à prendre dans les deux sens du terme - leur désinvolture pédante et ironique. Marc, Jean-Georges et les autres membres de leur bande, s'alignent dès lors sur la tradition du rejet du conformisme bourgeois : les règles ne leurs conviennent pas, et le style de vie qui les attend non plus. ${ }^{11}$ Cependant, si Kerouac rejetait les valeurs sociales de l'Amérique d'après-guerre, il vivait sans elles, tandis que Marc, tout en réprouvant cellesci, se nourrit des privilèges qu'elles lui octroient.

En ce temps-là, tout était grand. Nous passions nos journées dans de grandes écoles et nos nuits dans de grands appartements. [...] A force, nous étions tentés d'être des gagne-petit. [...] Bref il n'y avait pas de quoi se plaindre. ${ }^{12}$

Là, pourrait-on pointer, est le commencement de l'ambiguïté du cynisme de Marc. Il rejette tout en savourant les problèmes de la société française. C'est probablement pour cela qu'il tend à vouloir être célèbre : pour ne pas avoir à suivre les règles, que la population se doit de respecter, tout en ne

\footnotetext{
${ }^{10}$ Jack KEROUAC, Les anges vagabonds, Folio Gallimard, 1973, p. 4 de la partie 66.

${ }^{11}$ Frédéric BEIGBEDER, Mémoires d'un jeune homme dérangé, la petite Vermillon, Editions La Table Ronde, 2001, p. 33-34 et 66.

${ }^{12}$ Ibid., p. 13-14.
} 
devant pas se justifier. ${ }^{13}$ Oscar, un autre alter-ego de Beigbeder dans l'Egoiste romantique, veut parvenir à la renommée pour profiter de ses avantages : «Quand je m'enrhume, les gens croient que j'ai sniffé de la drogue. On appelle cela avoir une réputation. $\gg^{14}$ Non seulement il est ravi de pouvoir profiter de sa condition d'écrivain connu pour prendre de la cocaïne sans se cacher, mais il est aussi fier que cette habitude le caractérise. Oscar, en ce sens, personnifie les incohérences de l'organisation sociale. Plus loin, il raille le paradoxe que certains de ces avantages provoquent :

Dès que quelqu'un devient riche, il ne paie plus rien. Il erre de buffets gratuits en soirées sponsorisées. On ne veut surtout pas que l'argent des riches soit dépensé parce que ce serait trop simple. ${ }^{15}$

Une certaine infatuation émane de cette appartenance à ceux qui ne sont pas la «masse ». C'est pourquoi, pour revenir à Marc, le concept de privilège est si important : il doit se rendre à la soirée inaugurale du nouveau club de la Madeleine, Les Chiottes, car c'est une soirée sur invitation de Joss, DJ surbooké de New-York à Tokyo, dont l'accès est réservé aux grands noms des nuits parisiennes. Il faut qu'il en fasse partie. Marc doit se rendre à cet évènement, qui promet d'être le lieu d'une compétition acharnée pour déterminer parmi les invités qui est le plus en marge. Puisque la mondialisation et les nouvelles technologies ont multiplié le nombre de célébrités, celles-ci doivent, du coup, elles aussi lutter pour être encore plus célèbres, ou du moins originales, si ce n'est réellement marginales.

Il faut être en marge pour se sentir soi, pour se sentir en vie, pour ne pas se noyer dans le quotidien et la masse ${ }^{16}$ - la terreur d'Octave, qui ne veut pas tomber dans l'ennui et la fadeur. Et ce qui l'aide, c'est la cocaïne, jusqu'à ce qu'il ait le cœur brisé, et qu'il décide de revenir à une vie dite normale : «Octave tente de rester concentré. Il faut tenir sans coco, accepter la réalité non boostée, il faut faire partie de la société, respecter les êtres, jouer le jeu. » ${ }^{17}$ C'est en découvrant sa personne en souffrance qu'Octave comprend qu'il

\footnotetext{
${ }^{13}$ Du moins, jusqu'à l'emprisonnement au début d'Un roman français, Grasset, 2009, lorsque le narrateur, redevenu Frédéric, voit la justice rattraper sa célébrité.

${ }^{14}$ Frédéric BEIGBEDER, L'Egoiste romantique, Folio, Gallimard, 2006, p. 63.

${ }^{15}$ Ibid., p. 97.

${ }^{16}$ Frédéric BEIGBEDER, 99 Francs, Folio, Gallimard, 2004, p. 203.

${ }^{17}$ Ibid., p. 134.
} 
est un être comme les autres, et que le chemin de la marginalité ne rend pas heureux.

Il balaye les concepts de quotidien et de solidarité sociétale puisqu'il rejette le concept même de vie en société « Je préfère être dégueulasse et libre, ouais, tu m'as bien entendu, qu'éthique et prisonnier. ${ }^{18}$ Octave Parango ne respecte pas les lois, ni les règles du savoir-vivre, ni les gens autour de lui ; après tout, il est riche et son métier est de laver le cerveau des gens à coup de slogans bien trouvés. "Ainsi va la grande chaine du mépris publicitaire : le réalisateur méprise l'agence, l'agence méprise l'annonceur, l'annonceur méprise le public, le public méprise son voisin. ${ }^{19}$ Le mépris n'est pas inhérent au seul personnage d'Octave, il appartient à tous ses collègues, et comme le suicide de Marc le montre, à beaucoup d'autres gens.

Nous avons tous été choqués par le suicide de Marc. Mais dire que son geste nous a surpris serait mentir. [...] Nous savions tous que Marc était stressé, nous sentions bien qu'il se débattait, nous nous abreuvions de son entrain factice et nous changions de sujet quand il parlait d'autodestruction. Nous refusions l'évidence : Marronnier était en train de se tuer et nous n'avions pas l'intention de le sauver. Nous organisions son enterrement avant même sa mort. ${ }^{20}$

Cette façon de présenter l'évènement tragique du suicide de Marc est révélateur de la société individualiste. Lorsque l'on parle de marginalisation, ou de personnage marginal, on imagine toujours une personne anticonformiste, différente, qui ne « rentre pas dans le moule » et qui n'est donc pas bien acceptée par le reste de la société. Ici, chacun apparait comme marginal, puisque chacun risque de pouvoir mourir sans que personne ne l'en empêche, et sans que personne ne soit triste - le ton de ce paragraphe n'est pas lyrique, mais seulement, si l'on est optimiste, sympathique.

Chaque individu dans la société de l'individualisme est marginal puisque chacun veut trouver sa place « unique et à part » c'est-à-dire sans que celle-ci ne soit la même que celle d'un autre ; cette bataille perpétuelle pour être quelqu'un développe un égo, qui, s'il grandit trop met à l'écartconsciemment, comme dans le cas de Marc qui veut être connu pour ensuite ne plus vendre de livre ${ }^{21}$ ou plus inconsciemment, comme dans celui d'Octave qui

\footnotetext{
${ }^{18}$ Ibid., p. 203.

${ }^{19}$ Ibid., p. 232.

${ }^{20}$ Ibid., p. 179.

${ }^{21}$ Frédéric BEIGBEDER, Vacances dans le coma, Nouvelle Edition, Le livre de Poche, 2004.
} 
ne peut s'empêcher de trouver le bonheur au sein de jeunes filles prépubères qui le vénèrent. ${ }^{22}$ Puisque la société rend égocentré, il n'y a qu'un pas, rapidement et impunément franchi par les personnages beigbederiens, pour devenir irrespectueux et opportunistes :

La justification artistique autorisait toutes les expériences. Elles adoraient se sentir dédouanées. Je fournissais la caution culturelle, elles me prêtaient leur fente : on était plus proche du troc que du harcèlement. ${ }^{23}$

Le vocabulaire montre le peu de considération qu'a Octave pour les jeunes filles, et le capitalisme libéral lui permet d'excuser son abus de leur naïveté et de leur corps. Octave rejette les valeurs de respect de la société tout en absolvant son action par les valeurs de commerce de celle-ci : la faille est révélée, le cynisme a fonctionné.

Il est intéressant de mûrir cette idée en gardant en tête que ces personnages, Marc, Oscar et Octave, sont les alter-egos de leur auteur. Frédéric Beigbeder, qui fut chroniqueur mondain, organisateur du Caca's club, écrivain, publicitaire... est lucide lorsqu'il décrit ces différents mondes, connus de peu d'individus. Cette clairvoyance le place, une nouvelle fois, en marge de la société : faisant partie d'un groupe marginal, il doit de nouveau s'extraire pour en apprécier, avec subtilité, les trésors et les déboires. Ainsi donc, le travail de Beigbeder semblerait-il s'appliquer à une double forme de marginalité, laissant libre cours à son cynisme.

En effet, comme nous le rappelions précédemment, le cynique doit s'extraire, se mettre en surplomb du monde pour exercer sa sagacité et sa capacité à dénoncer les failles de la société. Comme le faisait Diogène, dit le chien, en Grèce antique, Beigbeder a recours à la provocation pour exprimer ses opinions.

"Le monde entier est prostitué. Payer ou être payé, telle est la question". ${ }^{24}$ Cette phrase est capitale ; le lecteur sera d'abord choqué puis refusera le propos séditieux. Ce n'est qu'en la méditant, qu'il pourra être convaincu. En effet, si l'on considère que la prostitution est la marchandisation du corps et de ses capacités, dès lors tout métier est prostitution. Une irrévérence provient

\footnotetext{
22 « Dans ses incarnations précédentes, le narrateur était odieux dans un périmètre restreint; grâce à l'invention du fascisme cosmétique féminin universel, il est devenu une sorte de haut dignitaire du régime, a la capacité de nuisance beaucoup plus redoutable. » Marin de Viry, Préface d'Au secours pardon, Le Livre de Poche, 2008, p. 13.

${ }^{23}$ Frédéric BEIGBEDER, Au secours pardon, Le Livre de Poche, 2008, p. 216.

${ }^{24}$ Frédéric BEIGBEDER, 99 Francs, Folio, Gallimard, 2004, p. 187.
} 
aussi de l'intertextualité de ce commentaire ; il reprend en effet le « To be or not to be » de Shakespeare, en le mettant au goût du jour, à la mode de la mondialisation et du sur consumérisme.

Lorsque l'on provoque, on s'expose à son audience, et de ce fait il est possible soit de créer le lien, si l'appel est accepté ou de construire un mur, si la provocation est rejetée ; l'on peut choquer un lecteur, qui ensuite adhérera à l'idée, ou refusera de la considérer. Le pari de la provocation est un pari risqué. Beigbeder le choisit, et de ce fait se singularise. Tout au long de ses livres, le lecteur est confronté à des commentaires, prenant la forme d'accroches, déroutants : «Les bombes, je les préfère sexuelles, et les attentats, à la pudeur $»,{ }^{25}$ «Les filles qui font consommer les femmes sont celles qui excitent leur mari », ${ }^{26}$ « Toujours pas pris en otage dans ce pays musulman. Cela va finir par devenir vexant $»,{ }^{27}$ « Je cherche des raisons de me plaindre mais je n'en trouve pas. Ce qui m'en fournit une. $»^{28}$, chacun dénonçant un travers ou un problème de la société contemporaine : la violence, la surconsommation, le racisme, l'égocentrisme...

Le cynisme de Beigbeder tend à une double excommunication et permet dès lors à ces romans de cruellement dénoncer les failles de notre société moderne.

\section{Le rejet de soi}

Dans cette deuxième partie nous allons aborder la marginalisation comme rejet de soi, souvent associé à une estime de soi mal placée. Cependant, avant de poursuivre, nous souhaitons préciser que, même si nous dissertons sur des personnages principaux comme alter-ego de Frédéric Beigbeder, nous n'entendons pas juger - que ce soit moralement ou philosophiquement - la vie de cet auteur. Nous commenterons ce qu'il est possible de déduire des mots choisis dans ses romans.

Nous évoquions précédemment la position de mépris d'Octave vis-à-vis des consommateurs qu'il manipule avec les slogans et scénarios publicitaires qu'il écrit; nous allons reprendre cette idée, en nous concentrant cette fois sur Octave uniquement, et sa relation à lui-même. Octave est lucide ; bien qu'il se

\footnotetext{
${ }^{25}$ Frédéric BEIGBEDER, Au secours pardon, Le Livre de Poche, 2008, p. 136.

${ }^{26}$ Ibid., p. 31.

${ }^{27}$ Frédéric BEIGBEDER, L'Egoiste romantique, Folio, Gallimard, 2006, p. 95.

${ }^{28}$ Ibid., p. 91.
} 
pense au-dessus de la masse puisqu'il fait partie de la poignée de gens qui la contrôle, il est lui aussi sujet aux mêmes problèmes :

Tout est provisoire : l'amour, l'art, la planète Terre, vous, moi. [...]

Tout s'achète : l'amour, l'art, la planète Terre, vous, moi. [...]

Tout est provisoire et tout s'achète. L'homme est un produit comme les autres avec une date de limite de vente. Voilà pourquoi j'ai décidé de prendre ma retraite à 33 ans. C'est, parait-il, l'âge idéal pour ressusciter. ${ }^{29}$

Dans le premier chapitre de 99 Francs, Octave présente la société dans laquelle il vit pour en venir, par le biais d'une gradation inversée - un resserrement d'objet - à se présenter lui-même. D'un concept, général et abstrait par définition, il saute à lui-même, et se compare alors au fils de Dieu. Le pacte autofictionnel suit à la p. suivante ; c'est dit, Octave se croit au-dessus du monde. Il est le fils du nouveau Dieu : la consommation. Avec ses slogans publicitaires il diffuse les nouveaux idéaux de la religion du $21^{\text {ème }}$ siècle. Pour se faire, il doit être au-dessus, à part, il doit s'extraire de ce monde pour le voir tel qu'il est. C'est d'ailleurs pourquoi il évoque la résurrection de Jésus : au milieu du roman - et de sa vie - Octave part en cure de réhabilitation. En un sens il disparait du monde et de sa société, pour revenir, différent, ayant compris qu'il faut que les choses changent. Octave sait qu'il sera congédié lorsque son livre sera publié, puisqu'il y dénonce les travers et corruptions du monde de la publicité. L'estime de soi, dans les romans de Beigbeder, va de pair avec l'autodestruction. Dans ses romans, un personnage masculin, sûr de lui et égoïste, évolue et mue, grâce à une lucidité sur sa vie et ce qui l'entoure, en un personnage qui, sinon s'engage, du moins sait qu'il faut agir pour changer les choses - que ce soit dans le domaine public ou privé.

L'estime de soi est un atout ; mais peut aussi être une entrave si elle est mal placée. Lorsque nous disons mal placée, nous faisons allusion à un surplus - l'égocentrisme - ou à un mensonge - que l'on se dit à soi-même. Dans les deux cas, cela peut conduire à une marginalisation, consciente ou inconsciente. Le premier cas fait rejeter l'autre et la société, le second a pour conséquence une éviction de soi-même, forme d'autodestruction. Les personnages beigbederiens sont toujours à la limite de ces deux options, puisqu'ils oscillent entre la crânerie, et un désespoir qui pointe sous la gaité :

\footnotetext{
${ }^{29}$ Frédéric BEIGBEDER, 99 Francs, Folio, Gallimard, 2004, p. 17-18.
} 
C'est un Narcisse complexé, pour résumer. Un type qui se regarde dans l'eau et qui recule, effrayé, plutôt que de se noyer en pamoison devant son image, comme dans la légende. Au lieu de se terminer tragiquement, la légende de Narcisse est détournée, et ne se termine jamais, comiquement. Narcisse revient au bord de l'eau tous les jours pour s'y mirer, et tous les jours, en se voyant, il crie « au secours je suis ignoble » et part en courant. Je suis ignoble physiquement et moralement mais je m'aime. ${ }^{30}$

Dans chaque processus établi par le personnage qui raconte son histoire, l'impulsion d'autodestruction domine : par exemple, dans Vacances dans le coma, Marc veut absolument avoir des relations sexuelles adventices même s'il est en couple avec Anne - comme le lecteur le découvre à la fin, après un retournement de situation comique ; dans 99 Francs, Octave écrit pour se faire virer ; dans Au secours pardon, il agit, regrette, se maudit et se confesse à un prêtre avant de se faire sauter avec la cathédrale ; dans L'Egoiste Romantique, Oscar dévoile dans son journal ses plus noirs secrets et dans Un roman français le narrateur consomme de la cocaïne sur une voiture, en pleine rue. Les antihéros de Beigbeder sont anti parce qu'ils s'aiment sans pouvoir se supporter. «Va, je ne te hais point, c'est moi que je déteste ; ma haine envers ma personne altère mes relations avec le reste du genre humain. Adieu, cageot de ma vie. ${ }^{31}$ Il rejette les filles laides pour ne pas se rappeler sa laideur, il rejette les failles de la société tout en les exploitant. Il refuse la société pour se rejeter lui-même.

J'aime répéter que ma bêtise est celle de mon temps mais au fond je sais que mon temps a bon dos et que ma bêtise m'appartient. A quarante balais, on est responsable de son malheur, même si l'on parait plus frais que lui. Ah oui, j'oublie de dire que j'ai quitté ma femme parce qu'elle avait le même âge que moi. $^{32}$

Après avoir récusé la société aussi cruellement qu'il le pouvait, Octave se regarde de nouveau et comprend que c'est lui qu'il doit rejeter. Il ne veut pas suivre les normes sociétales, et pourtant il subit les normes qu'il s'est créées. Dans L'Amour dure trois ans, Marc faisait le même constat. En ne voulant pas suivre les étapes habituelles des relations amoureuses il a perdu Anne, puis

\footnotetext{
${ }^{30}$ Marin de VIRY, préface d'Au secours pardon, Le Livre de Poche, 2008, p. 10.

${ }^{31}$ Frédéric BEIGBEDER, Au secours pardon, Le Livre de Poche, 2008, p. 57.

${ }^{32}$ Ibid., p. 61.
} 
Alice. Le roman finit bien parce qu'il tente - et réussit ? au moins pour un certain temps -à modifier ses propres règles. Il doit accepter de devenir normal.

Comment ai-je pu laisser les apparences dicter ma vie à ce point-là ? On dit souvent qu' «il faut sauver les apparences ». Moi je dis qu'il faut les assassiner car c'est le seul moyen d'être sauvé. ${ }^{33}$

Répéter souvent ces trois phrases:

1) LE BONHEUR N'EXISTE PAS.

2) L'AMOUR EST IMPOSSIBLE.

3) RIEN N'EST GRAVE. ${ }^{34}$

Marc Marronnier renonce à son pessimisme amoureux, et renonce au diktat des apparences.

Il est assez exaspérant de s'apercevoir que l'on a les mêmes interrogations que tout le monde. C'est une leçon de modestie..$^{35}$

Il doit accepter sa condition d'homme, d'être humain, qui partage de nombreuses caractéristiques avec les autres membres de son espèce.

On se marie exactement comme on passe son baccalauréat ou son permis de conduire : c'est toujours le même moule dans lequel on veut se couler pour être normal, normal, NORMAL, à tout prix. A défaut d'être au-dessus de tout le monde, on veut être comme tout le monde, par peur d'être en dessous. ${ }^{36}$

Si Marc doit faire la concession de ne pas vouloir être « au-dessus » à tout prix, il comprend aussi que la normalité peut être une référence, une aide, une protection : une société individualiste n'est pas uniquement composée de personnages nombrilistes, fiers et assurés. Marc découvre, comme Octave le fera par la suite, ses «blessures narcissiques $»^{37}$ lorsqu'il est confronté au rejet de celle qu'il aime. Une fois de plus, le rejet est au cœur de l'action du héros

\footnotetext{
${ }^{33}$ Frédéric BEIGBEDER, L'Amour dure trois ans, Folio, Gallimard, 2005, p. 23.

${ }^{34}$ Ibid., p. 33.

${ }^{35}$ Ibid., p. 84.

${ }^{36}$ Ibid., p. 48.

${ }^{37}$ Nous reprenons ici l'expression de Marin de VIRY, dans sa préface d'Au secours pardon, Le Livre de Poche, 2008, p. 11.
} 
beigbederien. Il rejetait la société sans éprouver le rejet de la société. Ressentir le rejet d'Alice fonctionne comme un double miroir.

Regardez-moi ce que je suis devenu... j'écris le même livre que les autres... Chassés croisés amoureux... On quitte une femme pour une autre qui ne vient pas... que m'arrive-t-il ? Où sont mes soirées décadentes ? [...] On dirait du jeune cinéma français... L'amour est le problème des gens qui n'ont pas de problèmes... Mais c'est la première fois que je ressens un pareil besoin physique d'écrire... [...] Peut-être n'est-il pas inepte de prendre son cas pour une généralité... Si je suis banal, alors je suis universel...Il faut fuir l'originalité, s'atteler aux sujets éternels... Marre du second degré... Je fais l'apprentissage de la sincérité... ${ }^{38}$

Marc fait l'apprentissage de la sincérité, après s'être détourné des apparences, pour apprendre la normalité. Il avoue, finalement, que même lui ne se considère pas comme exceptionnel, hors-normes; il se marginalisait pour se prouver qu'il était différent, original, mieux que le commun des mortels. Il se marginalisait pour se convaincre qu'il était mieux, qu'il était quelqu'un, et non pas seulement un membre de la masse. C'est en choisissant d'écrire l'histoire de son divorce et de sa rencontre avec Alice qu'il se rappelle qu'il est sujet, universellement, aux mêmes questionnements que les autres.

Nous touchons ici une ambiguité dans le rejet personnel du personnage beigbederien : il rejetait la société pour mieux se rejeter lui-même tout en voulant être rejeté par les autres sans être rejeté par l'Autre. Plus concrètement, Marc vit mal d'être rejeté par Alice dans L'Amour dure trois ans, mais il cherche aussi à être rejeté, dans Mémoires d'un jeune homme dérangé et dans Vacances dans le coma, son attitude, qui n'emprunterait ici de Diogène que les mots, indique cette envie, cette curiosité, cette volonté autodestructrice d'être rejeté. Pour satisfaire cette pulsion, il se met en danger.

C'est Robert qui engage la conversation.

- Tiens ? Voici ton ex-petit copain. Alors, il fait une pause ?

- Solange, ôte ton mec de mon soleil, tu veux ? S'écrie Marc. ${ }^{39}$

\footnotetext{
${ }^{38}$ Frédéric BEIGBEDER, L'Amour dure trois ans, Folio, Gallimard, 2005, p. 148.

${ }^{39}$ Frédéric BEIGBEDER, Vacances dans le coma, Nouvelle Edition, Le livre de Poche, 2004, p. 121.
} 
Robert, le nouveau copain de Solange est plus costaud que Marc, qui se fera rouer de coups, pour le plaisir d'un bon mot, et le goût de la provocation. De la même manière, il cherche toute la soirée à draguer des filles, et ce n'est qu'à l'aube qu'il se rappelle de sa situation matrimoniale :

Tout s'éclaire soudain. Marc se souvient; il a l'air fin. Cette Anne, non seulement son visage ne lui est pas inconnu, mais en plus il l'a épousé voilà deux ans. L'ivresse l'avait troublé : Marc vient de passer la nuit entière à chercher ce qu'il avait sous la main. ${ }^{40}$

Cependant, cette ambigüité est claire pour Marc ; il est conscient de vouloir être rejeté, il sait qu'il agit pour ne pas être accepté, car il ne veut pas être comme tout le monde.

Vous venez de mettre le doigt sur ma grande ambition : décevoir. Je m'efforce de décevoir le plus souvent possible. C'est la seule façon pour que les autres continuent de s'intéresser à moi. ${ }^{41}$

Il se marginalise pour être au centre de l'attention. Il rejette la société pour que celle-ci s'intéresse à lui ; il se rejette lui-même car il ne peut être d'accord avec ses normes ; mais celles-ci lui permettent d'accéder à la renommée qu'il convoite tant, tout comme Oscar dans L'Egoiste Romantique.

Nous avons vu que la marginalité chez Beigbeder est double ; elle est rejet de la société et rejet de soi-même, elle est voulue et inconsciente, elle est estime de soi et autodestruction. Mais, cette marginalité choisie trouve ses limites lorsqu'elle ne peut pas se différencier assez radicalement de la marginalité acceptée. Lorsqu'on fait l'objet d'un rejet, et que, pour l'accepter, on cherche alors à se retrancher dans le détail qui motive le rejet, on l'exagère et on exacerbe bien souvent cet écart par une sorte d'esprit de challenge. La marginalité d'un être n'est pas constante. Elle doit être cultivée, soit par ceux qui rejettent, soit par celui qui est rejeté.

Les personnages de Beigbeder semblent être dans un entre-deux. Ils se posent comme marginaux et en dehors de la société - la posture cynique - tout en faisant partie de cette même société, puisqu'ils en cultivent les avantages. Octave, dans Au secours pardon, se décrit alors comme le chat de Schrödinger, "à la fois vivant et mort.". En effet, le héros beigbederien semble être dans

\footnotetext{
${ }^{40}$ Ibid., p. 148.

${ }^{41}$ Ibid., p. 47.
} 
une situation paradoxale, puisqu'elle implique s'extraire tout en s'insérant. Ce paradoxe peut être expliqué par l'engouement, depuis les années 1990, de la société pour le cynisme, qui se manifeste dans cette attraction du mode de vie alternatif, cette volonté d'être soi, individu spécial au sein de la mondialisation.

En effet si l'on se réfère aux goûts des consommateurs - lecteurs - il faut remarquer que l'ancrage dans la réalité et la plongée dans le bouillonnement sociétal sont accrocheurs : les deux livres de Beigbeder qui ont reçu le meilleur accueil commercial s'insèrent directement dans la réalité, 99 Francs et la dénonciation de la publicité, et Windows on the World, le roman qui invente les derniers instants des personnes présentes dans la tour nord newyorkaise le 11 septembre 2001. 